\title{
ON $p$-HYPONORMAL CONTRACTIONS
}

\author{
B. P. DUGGAL
}

(Communicated by Palle E. T. Jorgensen)

\begin{abstract}
The contraction $A$ on a Hilbert space $H$ is said to be $p$-hyponormal, $0<p<1$, if $\left(A^{*} A\right)^{p} \geq\left(A A^{*}\right)^{p}$. Let $A$ be an invertible $p$ hyponormal contraction. It is shown that $A$ has $C_{.0}$ completely nonunitary part. Now let $H$ be separable. If $A$ is pure and the defect operator $D_{A}=\left(1-A^{*} A\right)^{1 / 2}$ is of Hilbert-Schmidt class, then $A \in C_{10}$. Let $B^{*}$ be a contraction such that $B^{*}$ has $C_{.0}$ completely nonunitary part, $D_{B^{*}}$ is of Hilbert-Schmidt class, and $B^{*}$ satisfies the property that if the restriction of $B^{*}$ to an invariant subspace is normal, then the subspace reduces $B^{*}$. It is shown that if $A X=X B$ for some quasi-affinity $X$, then $A$ and $B$ are unitarily equivalent normal contractions.
\end{abstract}

\section{INTRODUCTION}

Let $H$ be a Hilbert space, and let $B(H)$ denote the algebra of operators, i.e., bounded linear transformations, on $H$ into itself. An operator $A \in B(H)$ is said to be hyponormal if $A^{*} A \geq A A^{*}$. The class of semihyponormal or $\frac{1}{2}$-hyponormal, operators was introduced by Xia [12] by weakening the hyponormality condition $A^{*} A \geq A A^{*}$ to $\left(A^{*} A\right)^{1 / 2} \geq\left(A A^{*}\right)^{1 / 2}$. More generally, $A \in B(H)$ is said to be $p$-hyponormal, $0<p<1$, if $\left(A^{*} A\right)^{p} \geq\left(A A^{*}\right)^{p}$. The class of $p$-hyponormal operators has been studied in a number of papers, mainly by Xia and Aluthge (see $[1,2,12,13]$ and some of the references in [2]). Although the class of $p$-hyponormal operators is independent of the class of hyponormal operators (there exist $p$-hyponormal operators which are not hyponormal $[1,2,13])$, it is nevertheless true that $p$-hyponormal operators share many properties with hyponormal operators (see [2] for some instances of this). In this note we consider invertible $p$-hyponormal contractions $A$, and show that (just as for hyponormal contractions) the completely nonunitary part of $A$ is of class $C_{.0}$. Also it will be shown that (in common with hyponormal contractions) if the Hilbert space $H$ is separable, $A$ is pure, and the defect operator $D_{A}$ is of Hilbert-Schmidt class, then $A \in C_{10}$. We also prove a Putnam-Fuglede theorem type commutativity result for $p$-hyponormal contractions.

Received by the editors November 5, 1991.

1991 Mathematics Subject Classification. Primary 47B10, 47B20, 47A10.

Key words and phrases. $p$-hyponormal contraction, Hilbert-Schmidt class, quasi-similar, $C_{0}$ contraction. 


\section{NOTATION AND TERMINOLOGY}

The closure of the range and the orthogonal complement of the kernel of an $X \in B(H)$ will be denoted by $\overline{\operatorname{ran} X}$ and $\operatorname{ker}^{\perp} X$, respectively. An $X \in B(H)$ is said to be a quasi-affinity if both $X$ and $X^{*}$ have dense range, and $A, B \in$ $B(H)$ are said to be quasi-similar if there exist quasi-affinities $X, Y \in B(H)$ such that $A X=X B$ and $B Y=Y A$. We shall denote the (open) unit disc (in the complex plane) by $D$, and the boundary of $D$ will be denoted by $\partial D$. The spectrum, the joint point spectrum, and the point spectrum of an $A \in B(H)$ will be denoted by $\sigma(A), \sigma_{j p}(A)$, and $\sigma_{p}(A)$. (The point $\lambda=r e^{i \theta}, r \geq 0$, is said to be in the joint point spectrum of $A \in B(H)$, where $A$ has polar decomposition $A=V|A|$, if there exist a common eigenvector $f \neq 0$ of $V$ and $|A|$ such that $V f=e^{i \theta} f$ and $|A| f=r f$.)

The contraction $A$ is said to be c.n.u. (= completely nonunitary) if there exists no nontrivial reducing subspace $H_{0}$ of $H$ such that $A \mid H_{0}$ is unitary. We say that the contraction $A$ is of class $C_{.0}, A \in C_{.0}$, if $A^{* n} \rightarrow 0$ strongly as $n \rightarrow \infty$ and that $A \in C_{1}$. if $\inf _{n}\left\|A^{n} x\right\|>0$ for all nonzero $x \in H$. The class $C_{\alpha \beta}$, for $\alpha, \beta=0,1$, is defined by $C_{\alpha \beta}=C_{\alpha .} \cap C_{. \beta}$. The c.n.u. contraction $A$ belongs to the class $C_{0}$ if there exists an inner function $\varnothing$ such that $\varnothing(A)=0$. Recall that if $A \in C_{0}$, then amongst all inner functions $\varnothing$ such that $\varnothing(A)=0$ there is a minimal one (i.e., one which is a divisor, in the Hardy space $H^{\infty}$, of all others) called the minimal function of $A$. The defect operator $D_{A}$ of the contraction $A$ is defined by $D_{A}=\left(1-A^{*} A\right)^{1 / 2} ; D_{A}$ is said to be of the Hilbert-Schmidt class if trace $D_{A}^{2}<\infty$.

\section{RESULTS}

Throughout the following $A$ will denote an invertible $p$-hyponormal contraction $(0<p<1)$ with polar decomposition

$$
A=V|A|, \quad V \text { unitary and }|A|>0 .
$$

The contraction $\widehat{A}$ will be defined by

$$
\widehat{A}=|A|^{1 / 2} V|A|^{1 / 2},
$$

and it will be assumed that $\widehat{A}$ has polar decomposition

$$
\widehat{A}=\widehat{V}|\widehat{A}| \text {. }
$$

The contraction $W$ will be defined by

$$
W=|\widehat{A}|^{1 / 2} \widehat{V}|\widehat{A}|^{1 / 2}
$$

Then $A$ is similar to $W$; and if $0<p<\frac{1}{2}$, then $W$ is hyponormal [2, Corollary 3.3].

Theorem 1. The c.n.u. part of $A$ is of class $C_{.0}$.

Proof. By Löwner's theorem [7] a $p$-hyponormal operator is $q$-hyponormal for $q \leq p$. Hence if $\frac{1}{2} \leq p<1$ and $A$ is $p$-hyponormal, then $A$ is $q$-hyponormal for $0<q<\frac{1}{2}$. Thus it is sufficient to prove the theorem for $0<p<\frac{1}{2}$. 
Suppose $A$ is c.n.u. Let $(0 \neq) y \in H$, and let $\left\{y_{n}\right\}$ be the sequence defined by $y_{n}=A^{* n} y$. Then $\left\{\left\|y_{n}\right\|\right\}$ is a monotonic decreasing bounded numerical sequence which converges to its greatest lower bound $p_{y}$ (say). We have two possibilities: either $p_{y}=0$ or $p_{y}>0$. If $p_{y}=0$ for all $y \in H$, then $A \in C_{.0}$ and we are done; if $p_{y}>0$, then let $M$ be the subspace

$$
M=\left\{y \in H: 0<p_{y} \leq\left\|A^{* n} y\right\|, n=0,1,2, \ldots\right\} .
$$

Then there exists a co-isometry $U$ and a quasi-affinity $X$ such that

$$
A X=X U
$$

(this follows from the proof of [8, Proposition II.5.3]). Define the quasi-affinity $S$ by $S=|\widehat{A}|^{-1 / 2} \widehat{V}^{*}|A|^{-1 / 2} V^{*} X$; then

$$
W S=S U,
$$

where $W$ is hyponormal. By the Putnam-Fuglede theorem for hyponormal $W$ and co-hyponormal $U$ applied to (3) we conclude that $W$ and $U$ are unitarily equivalent unitary operators [10]. Since $A$ is similar to $W, A$ is similar to a unitary operator, and so $\sigma(A) \subseteq \partial D$.

Recall that

$$
\|A\|=r_{\mathrm{sp}}(A) \quad(=\text { spectral radius of } A)
$$

$[2$, Theorem 3.1] and

$$
\left\|A^{-1}\right\|=\frac{1}{\min \{|z|: z \in \sigma(A)\}}
$$

[2, Theorem 3.12(3)]. Hence $\|A\|=\left\|A^{-1}\right\|=1$. We have

$$
\|A x\|^{k} \leq\|x\|^{k}=\|x\|\|x\|^{k-1}=\left\|A^{-k} A^{k} x\right\|\|x\|^{k-1} \leq\left\|A^{k} x\right\|\|x\|^{k-1}
$$

for given natural numbers $k \geq 2$ and all $x \in M$. Consequently, $A$ is $k$ paranormal. (An operator $T$ is said to be $k$-paranormal on $H$ if $\|T x\|^{k} \leq$ $\left\|T^{k} x\right\|\|x\|^{k-1}$ for all $x \in H$.) Since a $k$-paranormal contraction similar to a unitary is unitary [3, Corollary 1], $M$ reduces $A$ and $A \mid M$ is unitary-a contradiction. Hence $A \in C_{.0}$.

Corollary 1. If $A X B=X$ for some contraction $B^{*}$ with $C_{.0}$ c.n.u. part and operator $X$, then $\overline{\operatorname{ran} X}$ reduces $A, \operatorname{ker}^{\perp} X$ reduces $B^{*}$, and $A \mid \overline{\operatorname{ran} X}$ and $B^{*} \mid \operatorname{ker}^{\perp} X$ are unitarily equivalent unitary operators.

Proof. Since $A$ has $C_{.0}$ c.n.u. part, [4, Theorem 2(a)] applies.

It is immediate from Theorem 1 that the pure part of $A$ (i.e., the completely $p$-hyponormal part of $A$ ) has representation of type

$$
\left(\begin{array}{cc}
C_{00} & * \\
0 & C_{10}
\end{array}\right) \text {. }
$$

Takahashi and Uchiyama [11, Theorem 4] have shown that a pure hyponormal contraction $B$ (on a separable Hilbert space $H$ ) with the defect operator $D_{B}$ in the Hilbert-Schmidt class (denoted in the sequel by $C_{2}$ ) if of class $C_{10}$. That this result holds for $A$ such that $D_{A} \in C_{2}$ is the content of our next theorem. We shall assume henceforth that our Hilbert space $H$ is separable. 
Theorem 2. If $A$ is pure and $D_{A} \in C_{2}$, then $A \in C_{10}$.

Proof. We start by considering the case $0<p<\frac{1}{2}$. Then the contraction $\widehat{A}$ (of (1)) is $\left(p+\frac{1}{2}\right)$-hyponormal and so semihyponormal (= $\frac{1}{2}$-hyponormal) by Löwner's theorem. This implies

$$
\widehat{V}^{*}|\widehat{A}| \widehat{V} \geq|\widehat{A}| \geq \widehat{V}|\widehat{A}| \widehat{V}^{*}
$$

Set $T=V^{*}|A| V$ and $S=|A|$; then

$$
|\widehat{A}|^{2}=\widehat{A}^{*} \widehat{A}=|A|^{1 / 2} V^{*}|A| V|A|^{1 / 2}=S^{1 / 2} T S^{1 / 2} \geq S^{1 / 2+1 / 2+1}=S^{2}=|A|^{2}
$$

(see Furuta [6]). Hence

$$
|\widehat{A}|^{1 / 2} \widehat{V}^{*}|\widehat{A}| \widehat{V}|\widehat{A}|^{1 / 2} \geq|\widehat{A}|^{2} \geq|A|^{2}
$$

and

$$
\left(1-A^{*} A\right)=1-|A|^{2} \geq 1-|\widehat{A}|^{1 / 2} \widehat{V}^{*}|\widehat{A}| \widehat{V}|\widehat{A}|^{1 / 2}=1-W^{*} W \quad(\geq 0) .
$$

Since $D_{A} \in C_{2}, D_{W} \in C_{2}$. The contraction $W$ being hyponormal, and similar to $A$, is of class $C_{.0}$ (since $A$ is, by Theorem 1 ). Hence $W$ has direct sum decomposition $W_{0} \oplus W_{10}$, where $W_{0} \in C_{0}$ and $W_{10} \in C_{10}$ [11, Theorems 1 and 4]. Also, $W_{0}$ is normal, so that $\sigma\left(W_{0}\right)=\sigma_{p}\left(W_{0}\right) \subset D$ is countable [8, Theorem III.5.1].

Suppose $A$ has a nontrivial $C_{00}$ part. Then

$$
A=\left(\begin{array}{cc}
A_{0} & * \\
0 & A_{1}
\end{array}\right), \quad \text { where } A_{0} \in C_{00} \text { and } A_{1} \in C_{10} .
$$

The similarity of $A$ and $W$ implies the existence of invertible operators $Y=$ $\left[Y_{i j}\right]_{i, j=1}^{2}$ and $Z=\left[Z_{i j}\right]_{i, j=1}^{2}\left(Z^{-1}=Y\right)$ such that $A Y=Y W$ and $Z A=W Z$. Since $A_{1} Y_{21}=Y_{21} W_{0}$ and $Z_{21} A_{0}=W_{10} Z_{21}, Y_{21}=0=Z_{21}$. Thus $A_{0} Y_{11}=$ $Y_{11} W_{0}$ and $Z_{11} A_{0}=W_{0} Z_{11}$, where $Y_{11}$ and $Z_{11}$ are injective. Consequently, $A_{0}$ and $W_{0}$ are quasi-similar $C_{0}$ contractions [9, Theorem 1] and so have the same spectrum [8]. Thus $\sigma\left(A_{0}\right)=\sigma_{p}\left(A_{0}\right) \subset D$ is countable.

Let $m_{A_{0}}$ denote the minimal function of $A_{0}$. Then since $A_{1}$ has empty point spectrum, the point spectrum of $A$ consists of the zeros of $m_{A_{0}}$. Since $\sigma_{j p}(A)=\sigma_{p}(A)($ see $[13 ; 2$, Theorem 3.8]) and the eigenspaces of $V$ reduce $A$. (see [13;2, Theorem 3.5]), $A=A_{0} \oplus A_{1}$. We assert that $A_{0}$ is normal: this would then imply that $A$ could not have a nontrivial $C_{00}$ part and hence that $A \in C_{10}$ (completing thereby the proof for the case $0<p<\frac{1}{2}$ ).

Let $B=V\left|A_{0}\right|^{p}$; then $B$ is a hyponormal contraction with $\sigma(B)=\sigma_{p}(B)=$ $\tau\left(\sigma\left(A_{0}\right)\right)=\tau\left(\sigma_{p}\left(A_{0}\right)\right)$, where $\tau\left(r e^{i \theta}\right)=r^{p} e^{i \theta}$ [2, Theorem 3.14]. Since $\sigma(B)=$ $\sigma_{p}(B)$ is countable, $\sigma(B)$ has zero Lebesgue area measure, implying thereby that $B$ is normal. Thus

$$
0=B^{*} B-B B^{*}=\left(A_{0}^{*} A_{0}\right)^{p}-\left(A_{0} A_{0}^{*}\right)^{p},
$$

i.e., $A_{0}$ is normal.

To complete the proof we note (as before) that if $A$ is $p$-hyponormal, $\frac{1}{2} \leq$ $p<1$, then $A$ is $q$-hyponormal for $q \leq p$. Hence the argument above applies.

We prove now a Putnam-Fuglede theorem for $p$-hyponormal contractions. It will be assumed in the following theorem that the contraction $B^{*}$ satisfies the property: If the restriction of $B^{*}$ to an invariant subspace is normal, then the subspace reduces $B^{*}$. 
Theorem 3. If $A X=X B$ for some quasi-affinity $X$ and contraction $B^{*}$, with $C_{.0}$ c.n.u. part, satisfying $D_{B^{*}} \in C_{2}$, then $A$ and $B$ are unitarily equivalent normal contractions.

Proof. Let $S$ be the quasi-affinity $S=|\widehat{A}|^{-1 / 2} \widehat{V}^{*}|A|^{-1 / 2} V^{*} X$. Then, with $W$ as defined in (2), $W S=S B$. Since $W$ is hyponormal, $W$ and $B$ are unitarily equivalent normal contractions of type unitary $\bigoplus C_{0}$ (apply [5, Theorem $\left.1^{\prime}\right]$ ). Since $A$ is similar to $W, A Y=Y W$ for some invertible operator $Y$. We show that $A$ has no pure part. For suppose that $A$ has the direct sum decomposition $A=A_{n} \oplus A_{c}$, where $A_{n}$ is normal and $A_{c} \in C_{.0}$ is pure. Decompose $W$ by $W=W_{u} \oplus W_{0}$, where $W_{u}$ is unitary and $W_{0} \in C_{0}$ is normal, and let $Y$ have the corresponding matrix representation $Y=\left[Y_{i j}\right]_{i, j=1}^{2}$. Then, since $A_{c} Y_{21}=Y_{21} W_{u}, Y_{21}=0$. We have $A_{c} Y_{22}=Y_{22} W_{0}$ or $A_{c}\left(Y_{22} \mid \operatorname{ker}^{\perp} Y_{22}\right)=$ $\left(Y_{22} \mid \operatorname{ker}^{\perp} Y_{22}\right)\left(W_{0}^{*} \mid \operatorname{ker}^{\perp} Y_{22}\right)^{*}$. Since $Y_{22}$ has dense range and $W_{0} \in C_{0}$ is normal, $A_{c} \in C_{0}$ and $\sigma\left(A_{c}\right)=\sigma_{p}\left(A_{c}\right) \subset D$ is countable. Since $A_{c}$ is $p$ hyponormal, an argument similar to that used in the proof of Theorem 2 shows that $A_{c}$ is normal一a contradiction. This completes the proof.

Recall that given a $C_{10}$ contraction $B^{*}$ there exists an isometry $L$ and a quasi-affinity $Z$ satisfying $L Z=Z B^{*}$. Hence if $A X=X B, B^{*} \in C_{10}$, for some quasi-affinity $X$, then $A X Z^{*}=X Z^{*} L^{*}$. Applying Theorem 3 it follows that $A$ is unitarily equivalent to a unitary operator. Hence

$$
\|X x\|=\left\|A^{n} X x\right\|=\left\|X B^{n} x\right\| \leq\|X\|\left\|B^{n} x\right\| \rightarrow 0 \text { as } n \rightarrow \infty
$$

for all $x \in H$. We have:

Corollary 2. There exists no quasi-affinity $X$ such that $A X=X B$ for some $C_{10}$ contraction $B^{*}$.

\section{ACKNOWLEDGMENT}

It is my pleasure to thank Dr. Ariyadasa Aluthge for supplying me with a copy of [2].

\section{REFERENCES}

1. A. Aluthge, On p-hyponormal operator for $0<p<1$, Integral Equations Operator Theory 13 (1990), 307-315.

2. __ Properties of p-hyponormal operators, unpublished dissertation, Vanderbilt University, Nashville, TN, August 1990.

3. B. P. Duggal, Contractions with a unitary part, J. London Math. Soc. 31 (1985), 131-136.

4. __, On intertwining operators, Monatsh. Math. 106 (1988), 139-148.

5.

6. T. Furuta, $A \geq B \geq 0$ assures $\left(B^{r} A^{p} B^{r}\right)^{1 / q} \geq B^{(p+2 r) / q}$ and $A^{(p+2) / q} \geq\left(A^{r} B^{p} A^{r}\right)^{1 / q}$ for $r \geq 0, q \geq 1$, with $(1+2 r) q \geq(p+2 r)$, Proc. Amer. Math. Soc. 101 (1981), 85-88.

7. K. Löwner, Uber monotone matrix functionen, Math. Z. 38 (1934), 177-216.

8. B. Sz.-Nagy and C. Foiaş, Harmonic analysis of operators on Hilbert space, North-Holland, Amsterdam, 1970.

9. $\ldots$, On injections intertwining operators of class $C_{0}$, Acta Sci. Math. (Szeged) 40 (1978), 163-167.

10. J. G. Stampfli and B. L. Wadhwa, An asymmetric Putnam-Fuglede theorem for dominant operators, Indiana Univ. Math. J. 25 (1976), 359-365. 
11. K. Takahashi and M. Uchiyama, Every $C_{00}$ contraction with Hilbert-Schmidt defect operator is of class $C_{0}, \mathrm{~J}$. Operator Theory 10 (1983), 331-335.

12. D. Xia, On the non-normal operators-semihyponormal operators, Sci. Sinica 23 (1980), 700-713.

13. __ Spectral theory of hyponormal operators, Birkhäuser Verlag, Boston, 1983.

Department of Mathematics, National University of Lesotho, P.O. Roma 180 , LESOTHO, SOUTHERN AFricA

Current address: Department of Mathematics, Sultan Qaboos University, College of Science, P.O. Box 36, Al Khod 123, Oman 